\title{
Quality of life outcomes for brain metastasis patients treated with stereotactic radiosurgery: pre-procedural predictive factors from a prospective national registry
}

\author{
Jason P. Sheehan, MD, PhD, ${ }^{1}$ Inga Grills, MD, ${ }^{5}$ Veronica L. Chiang, MD, ${ }^{3}$ Huamei Dong, PhD, ${ }^{2}$ \\ Arthur Berg, PhD, ${ }^{2}$ Ronald E. Warnick, MD, ${ }^{7}$ Douglas Kondziolka, MD, MS,${ }^{4}$ and \\ Brian Kavanagh, MD, MPH ${ }^{6}$
}

\begin{abstract}
1Department of Neurological Surgery, University of Virginia, Charlottesville, Virginia; ${ }^{2}$ Penn State Medical Center, Hershey, Pennsylvania; ${ }^{3}$ Department of Neurological Surgery, Yale University, New Haven, Connecticut; ${ }^{4}$ Department of Neurological Surgery, New York University, New York, New York; ${ }^{5}$ Department of Radiation Oncology, Beaumont Health System, Royal Oak, Michigan; ${ }^{6}$ Department of Radiation Oncology, University of Colorado, Denver, Colorado; and ${ }^{7}$ Mayfield Clinic, Cincinnati, Ohio
\end{abstract}

OBJECTIVE Stereotactic radiosurgery (SRS) is increasingly used for the treatment of brain metastasis. To date, most studies have focused on survival, radiological response, or surrogate quality endpoints such as Karnofsky Performance Scale status or neurocognitive indices. The current study prospectively evaluated pre-procedural factors impacting quality of life in brain metastasis patients undergoing SRS.

METHODS Using a national, cloud-based platform, patients undergoing SRS for brain metastasis were accrued to the registry. Quality of life prior to SRS was assessed using the 5-level EQ-5D (EQ5D-L) validated tool; additionally, patient and treatment attributes were collected. Patient quality of life was assessed as part of routine follow-up after SRS. Factors predicting a difference in the aggregate EQ5D-L score or the subscores were evaluated. Pre-SRS covariates impacting changes in EQ5D-L were statistically evaluated. Statistical analyses were conducted using multivariate linear regression models.

RESULTS EQ5D-L results were available for 116 patients. EQ5D-L improvement (average of 0.387) was noted in patients treated with earlier SRS ( $p=0.000175$ ). Worsening overall EQ5D-L (average of 0.052 per lesion) was associated with an increased number of brain metastases at the time of initial presentation $(p=0.0399)$. Male sex predicted a risk of worsening (average of 0.347 ) of the pain and discomfort subscore at last follow-up ( $p=0.004205)$. Baseline subscores of pain/discomfort were not correlated with pain/discomfort subscores at follow-up ( $p=0.604)$, whereas baseline subscores of anxiety/depression were strongly positively correlated with the anxiety/depression follow-up subscores $(p=0.0039)$.

CONCLUSIONS After SRS, quality of life was likely to improve in patients treated early with SRS and worsen in those with a greater number of brain metastases. Sex differences appear to exist regarding pain and discomfort worsening after SRS. Those with high levels of anxiety and depression at SRS may benefit from medical treatment as this particular quality of life factor generally remains unchanged after SRS.

https://thejns.org/doi/abs/10.3171/2018.8.JNS181599

KEYWORDS brain metastasis; quality of life; stereotactic radiosurgery; oncology

$\mathrm{B}$ RAIN metastasis represents one of the most common tumors seen in adult patients. The incidence of brain metastases ranges from 8.3 to 14.3 per 100,000 patients, and following the initial diagnosis, many patients will develop additional brain metastasis within the course of their disease.$^{10}$ Goals of care for brain metas- tasis patients frequently place a premium on quality of life (QOL). However, most studies evaluate progression-free survival as the primary endpoint. 5,7,20,22

Stereotactic radiosurgery (SRS) is utilized with increasing frequency for brain metastasis patients. It has been shown to offer a high rate of local tumor control and

\footnotetext{
ABBREVIATIONS ASTRO = American Society for Radiation Oncology; BRCS = brain cancer subscale; EORTC = European Organisation for Research and Treatment of Cancer; EQ5D-L = 5-level EQ-5D; FACT-BR = Functional Assessment of Cancer Therapy-Brain; HRQOL = health-related QOL; KPS = Karnofsky Performance Scale; NPA = NeuroPoint Alliance; QOL = quality of life; SRS = stereotactic radiosurgery; WBRT = whole-brain radiotherapy.

SUBMITTED June 21, 2018. ACCEPTED August 3, 2018.
}

INCLUDE WHEN CITING Published online December 21, 2018; DOI: 10.3171/2018.8.JNS181599. 
typically avoids treatment-related neurocognitive decline that has been associated with whole-brain radiotherapy (WBRT). ${ }^{4,22}$ Compared to resection, radiosurgery can be performed in the ambulatory setting for most patients and avoids appreciable interruption of systemic therapy. Radiosurgery also offers durable control of most intracranial tumors well beyond the median survival for brain metastasis. ${ }^{4,22}$

Few studies to date have examined the changes in QOL for patients treated with SRS for brain metastasis. ${ }^{11,16}$ In the current study, we examine prospective QOL outcomes in brain metastasis patients treated with SRS. Patients were accrued as part of the national SRS registry for the American Association of Neurological Surgeons (AANS) and American Society for Radiation Oncology (ASTRO). The validated 5-level EQ-5D (EQ5D-L) assessment tool was used to assess patients prior to SRS and then at follow-up appointments longitudinally thereafter. ${ }^{14}$ EQ-5D differences following SRS and pre-SRS factors associated with these differences were examined in registry patients.

\section{Methods}

\section{Registry Initiative}

As previously described, the NeuroPoint Alliance (NPA), in conjunction with physicians from the AANS and ASTRO, created a national quality registry for SRS. ${ }^{15}$ The registry's primary focus was quality of care and, therefore, it was deemed institutional review board (IRB) exempt by the Western IRB and by the IRBs of participating centers.

\section{Radiosurgical Selection and Treatment}

From a total of 24 centers, the patients were accrued to the registry in a consecutive fashion at the time of SRS treatment. Selection of brain metastasis patients for SRS was as per the institutional protocol. Radiosurgery was delivered using various devices that were present at the accruing site, and these included linear accelerator-based SRS systems such as BrainLab's Novalis or the Gamma Knife. Dose planning was generally performed in a multidisciplinary fashion with the input of a neurological surgeon, radiation oncologist, and medical physicist. The dose selection, critical structure constraints, and fractionation scheme, if any, were decided upon by the local treating physicians.

\section{Study Patient Cohort and Data Collection}

Patients were accrued in a prospective fashion, and patient, clinical, and radiological responses were assessed and collected in a longitudinal fashion from the time of SRS. Using a common data dictionary and online software platforms, data were uploaded by participating sites to a central repository. Data veracity was checked by software range checking and periodic queries by registry coordinators to clinicians and data-coordinating staff at individual sites. Data were then aggregated by the NPA. The data were extracted from the repository on December 13, 2017, and then sent to two biostatisticians (H.D. and A.B.) for analysis.

Inclusion criteria for this study were patients treated with SRS for the diagnosis of brain metastasis and EQ5D-
TABLE 1. Patient and treatment parameters for the study cohort

\begin{tabular}{lcc}
\hline \multicolumn{1}{c}{ Factor } & Value & SD \\
\hline Median age at SRS, yrs & 59 & 15.29 \\
\hline Sex, no. of patients & & \\
\hline Female & 73 & \\
\hline Male & 43 & \\
\hline Median follow-up, days & 105 & 92.7 \\
\hline Median EQ5D-L at SRS & 1 & 0.14 \\
\hline Median EQ5D-L at last follow-up & 0.86 & 0.165 \\
\hline SRS tumor margin dose, Gy & & \\
\hline Median & 21.5 & \\
\hline Mean & 22.1 & 5.85 \\
\hline SRS tumor volume, cm ${ }^{3}$ & & \\
\hline Median & 0.3 & \\
\hline Mean & 4.9 & \\
\hline Mean no. of lesions treated per patient & 2.3 & 1.68 \\
\hline No. of SRS fractions & & \\
\hline Median & 1 & \\
\hline Mean & 1.3 & 0.94 \\
\hline
\end{tabular}

L testing shortly before the time of SRS and then EQ5D-L testing at least one time post-SRS. ${ }^{6}$ Patients were excluded from this analysis if they had an SRS indication in the registry that was something other than brain metastasis or if they had no pre-SRS or post-SRS EQ5D-L score.

Data fields collected included patient attributes, tumor and treatment variables, and EQ5D-L at SRS and followup points, and these are detailed in Table 1. Time from diagnosis of brain metastasis to initial SRS in days was evaluated as a continuous variable. Early SRS was defined as SRS within 60 days of initial brain metastasis diagnosis. Pre-SRS attributes were evaluated for their effect on overall EQ5D-L and subscores of EQ5D-L at last followup. The follow-up interval was as per the discretion of the treating physicians' recommendations and the clinical needs of the treated patient.

\section{Participating Sites}

At the time of the data cut from the registry, the following institutions were participating sites in the registry and had contributed patient data for the study purposes: University of Virginia, William Beaumont Hospital, New York University, Yale University, University of Colorado, Vanderbilt University, Duke University, University of California Los Angeles Medical Center, Carolinas Medical Center, University of Florida Cancer Center at Orlando Health, Penn State Medical Center, University of Rochester Medical Center, Mayfield Clinic, Jefferson Hospital, University of Utah, Valley Hospital of New Jersey, Semmes Murphey neurosurgical group, NewYork-Presbyterian/Weill Cornell Medical Center, and University of Southern California in Los Angeles.

\section{Statistical Methods}

Utilizing pre-SRS attributes, changes in EQ5D-L 
scores and dimension subscores were analyzed with a multivariate linear regression model following a reproducible statistical workflow using R v3.4.3 (https:/Www.Rproject.org) and R Markdown v1.8 (https:/CRAN.Rproject.org/package $=$ rmarkdown). All patients with one or more metastatic tumors at the time of index SRS and with QOL evaluations were included. A multiple-record data set was created with one record per follow-up visit distinguished by the time in days post-SRS of the record. Changes in the EQ5D-L score and subscores were modeled with covariate predictors of age, sex, diagnosis history, follow-up time, time from initial diagnosis to SRS, preSRS (i.e., neoadjuvant) brain metastasis resection, number of lesions, and log total tumor volume. Separate models analyzed the total score and each of the 5 subscores. Additional models considered the effect of baseline EQ5D-L scores on subsequent follow-up scores. ${ }^{8}$

\section{Results}

\section{Patient and Treatment Attributes}

One hundred sixteen brain metastasis patients who had EQ5D-L data prior to SRS and at follow-up as well were included in the study. The patient and treatment attributes are detailed in Table 1. Gamma Knife was used to treat $64.5 \%$ of patients, whereas a linear accelerator was used to treat the remaining $35.5 \%$. Immobilization was performed with a rigid stereotactic frame in $59.1 \%$ of cases and maskbased systems in the remaining $40.9 \%$ of cases. The median age was 59 years (SD 15.29 years). Median follow-up was 105 days (SD 92.7 days). The median EQ5D-L total scores at initial SRS and last follow-up were 1 and 0.86 , respectively.

For the brain metastasis patients included in the study, the primary cancer histology was $52.9 \%$ lung, $13.3 \%$ melanoma, $14.2 \%$ breast, and $5.1 \%$ kidney, and in the remaining patients the cancer histologies comprised more rare types (e.g., gastrointestinal, genitourinary). The mean SRS margin dose was 22.1 Gy, and the mean number of lesions treated per patient was 2.3. The mean number of fractions per SRS was 1.3.

\section{QOL Changes and Pre-SRS Factors Impacting QOL}

One hundred sixteen brain metastasis patients had EQ5D-L data prior to SRS and at follow-up as well as pre-SRS covariate data during the time of the data cut. At last follow-up, 49 patients $(42 \%)$ exhibited a decrease in the aggregate EQ5D-L score from the time of initial SRS. Covariates collected as part of the registry and included in the analysis of EQ5D-L differences included age, histological diagnosis, length of follow-up, sex, age at initial diagnosis, neoadjuvant (i.e., pre-SRS) resection, and total brain metastasis volume (Table 2). The change in EQ5D-L score for individual patients as recorded at each time point after initial SRS is depicted by the scatterplot in Fig. 1 upper. Differences in overall EQ5D-L scores for the entire cohort from pre-SRS EQ5D-L score to EQ5D-L at last follow-up are depicted in Fig. 1 lower.

Stability or improvement in overall EQ5D-L score at last follow-up was statistically associated with earlier use of SRS from the time of initial brain metastasis diagnosis
TABLE 2. Multivariate analysis of factors associated with differences in overall EQ5D-L scores

\begin{tabular}{lc}
\hline \multicolumn{1}{c}{ Prognostic Factor } & $p$ Value \\
\hline Age & 0.100000 \\
\hline Initial cancer diagnosis to brain metastasis dates & 0.979000 \\
\hline Histological diagnosis & 0.150000 \\
\hline Follow-up length & 0.378000 \\
\hline Male sex & 0.403000 \\
\hline Neoadjuvant (prior to SRS) resection & 0.138000 \\
\hline No. of brain metastases at initial diagnosis & 0.039900 \\
\hline Log (total brain metastasis volume) & 0.536000 \\
\hline $\begin{array}{c}\text { Early use of SRS from the time of initial brain } \\
\text { metastasis diagnosis }\end{array}$ & 0.000175 \\
\hline
\end{tabular}

$(\mathrm{p}=0.000175)$. Worsening overall EQ5D-L score at last follow-up was associated with increased number of brain metastases at the time of initial presentation of intracranial disease $(\mathrm{p}=0.0399)$. Regarding the impact of other covariates on the difference in EQ5D-L before and after SRS, age $(p=0.100)$, sex $(p=0.403)$, and length of follow-up $(p=0.378)$ were not significantly associated with changes in EQ5D-L scores at last follow-up.

\section{QOL Subscores and Pre-SRS Factors Impacting QOL Subscores}

Differences in pain and discomfort subscores of EQ5DL from time of initial SRS to last follow-up are depicted in Fig. 2. Multivariate analysis of factors affecting differences in pain and discomfort subscores of EQ5D-L were evaluated, and male sex $(p=0.004205)$ predicted a decrease (i.e., worsening) of the pain and discomfort subscore by 0.35 .

Differences in anxiety and depression subscores of the EQ5D-L from the time of initial SRS to last follow-up are depicted in Fig. 3. For the anxiety and depression subscore at baseline just prior to SRS, this was statistically predictive of the anxiety and depression subscore at last followup $(\mathrm{p}=0.00386)$ for brain metastasis patients.

\section{Discussion}

Survival is frequently used as the primary endpoint in brain metastasis studies. ${ }^{17,18,20,22}$ Nevertheless, there has been increasing interest in QOL as an indicator of outcome in studies of advanced cancer patients. ${ }^{2,18}$ In the EORTC (European Organisation for Research and Treatment of Cancer) phase III trial of adjuvant WBRT versus observation in patients with 1 to 3 brain metastases, healthrelated QOL (HRQOL) results were used as a secondary endpoint, and those patients in the observation-only arm (i.e., resection or SRS alone) demonstrated significantly higher HRQOL scores than did patients who underwent WBRT. ${ }^{17,18}$ Despite the widespread and growing use of SRS for brain metastasis, a recent Cochrane review of 5 randomized trials concluded that there was a relative lack of meaningful QOL outcomes data that could be derived from the use of SRS for brain metastasis patients. ${ }^{19,21}$

In a single-center study of 67 brain metastasis patients 

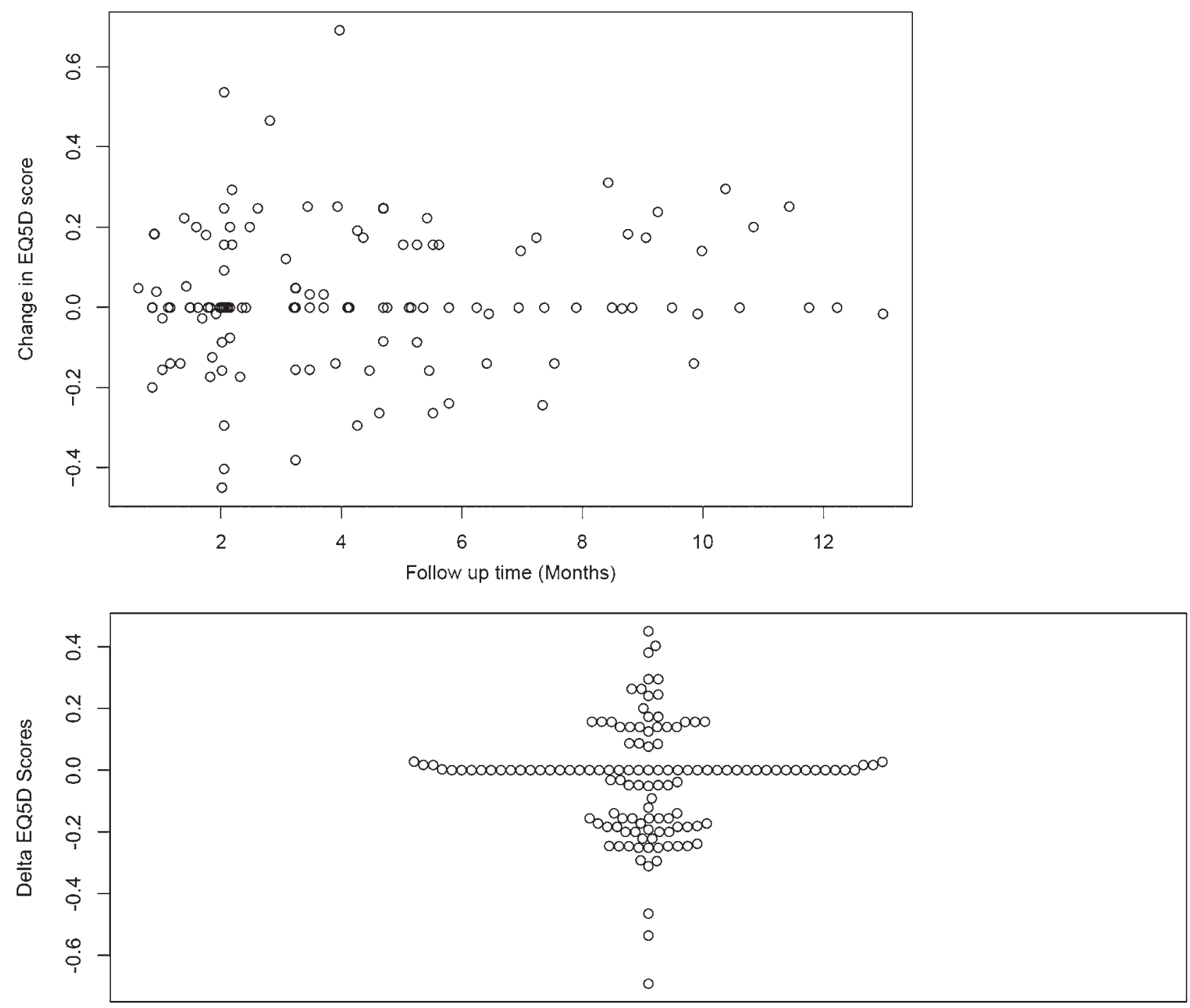

FIG. 1. Upper: Scatterplot of change in EQ5D-L score from time of initial assessment to the last follow-up time point for individual patients as recorded in the registry's database. Data are plotted as a function of the time elapsed from each individual patient's time of initial SRS. Lower: Differences in overall EQ5D-L score from the time of initial SRS to last follow-up for the overall cohort of 116 patients.

treated with SRS, 12-month EQ-5D index score preservation was $79 \%$. However, patients with more than 3 brain metastases had a greater deterioration in EQ-5D score than patients with only a single metastasis. ${ }^{9}$ In another study of 44 lung cancer brain metastasis patients treated with SRS, the Functional Assessment of Cancer Therapy-Brain (FACT-BR) was used to monitor QOL. ${ }^{3}$ In that study, the mean brain cancer subscale (BRCS) of the FACT-BR remained stable from baseline up to 1 year after SRS and improved for $72.3 \%$ of patients with a total brain metastasis volume of up to $5 \mathrm{~cm}^{3}$. Also, a higher or stable QOL was most predicted by a lower recursive partitioning analysis (RPA) classification, a higher Karnofsky Performance Scale (KPS) status, and an asymptomatic brain metastasis.

In an additional study of 97 brain metastasis patients treated with SRS who were assessed during follow-up by use of the BRCS of FACT-BR, 66\% of BRCS scores in patients were improved and $6 \%$ of patient scores were unchanged at 9 months post-SRS. ${ }^{16}$ High KPS score, asymptomatic brain metastasis, absence of cognitive deficits, and seizure freedom were predictors of high or stable BRCS at 12 months following SRS. In another study by Pan et al. (2008), decreased T1-weighted imaging and FLAIR volume $(\mathrm{p}<0.001)$ were associated with an improvement in the QOL of patients post-SRS as assessed by the Brain Cancer Module 20 questionnaire. ${ }^{11}$ Improved QOL postSRS was associated with extended overall survival in that same study. ${ }^{11}$

In the current study, age, sex, and total tumor volume at baseline were not associated with decreased QOL. This suggests that within the current contemporary practice of SRS for brain metastasis, these factors should not necessarily be used as a primary determinant of SRS eligibility, 


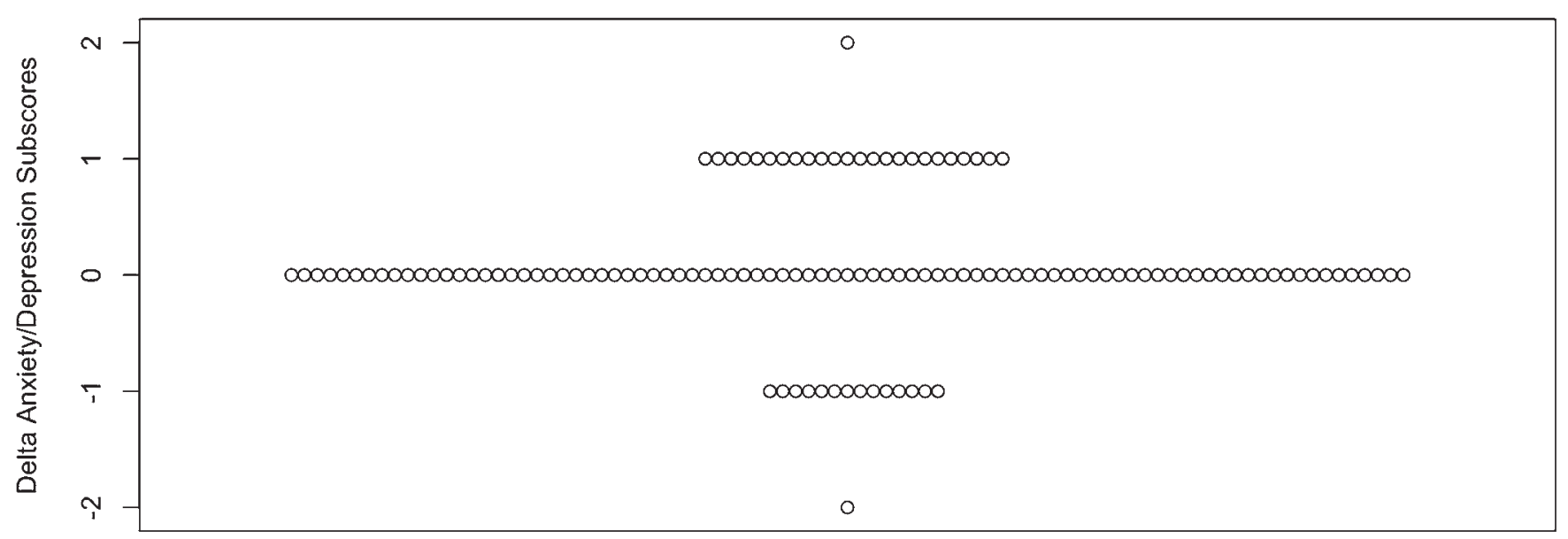

FIG. 2. Differences in EQ5D-L anxiety and depression subscores from the time of initial SRS to last follow-up for the overall cohort of 116 patients. Circles represent patients tested.

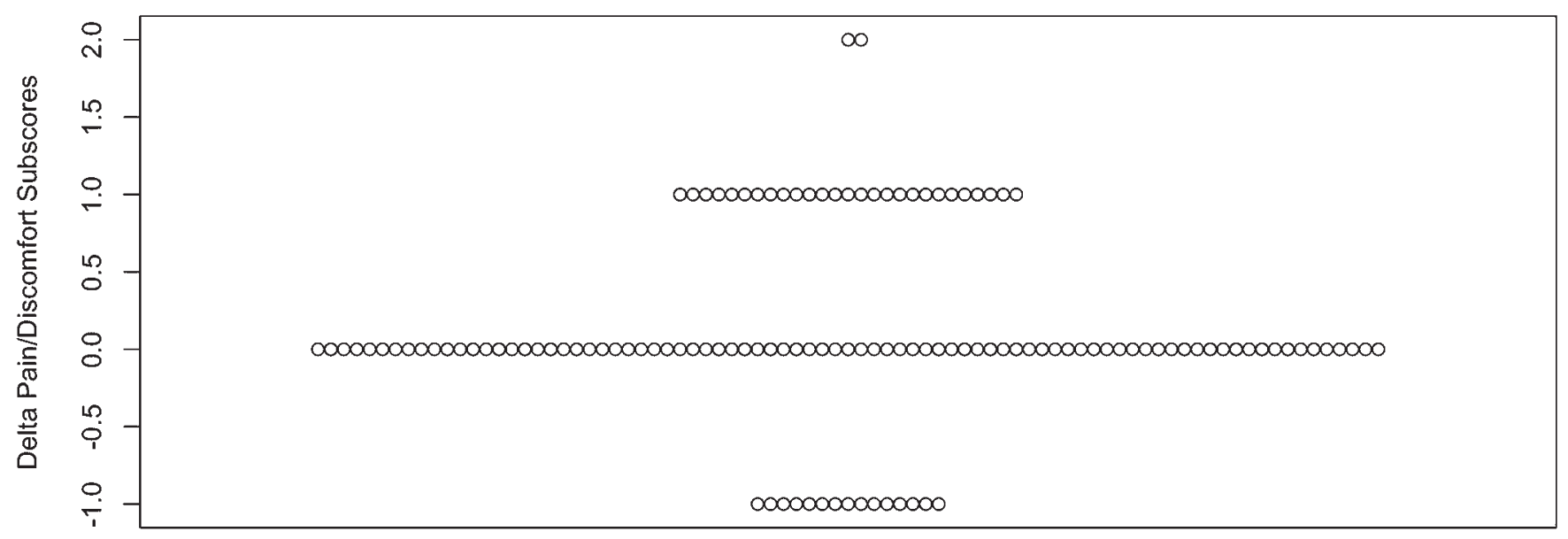

FIG. 3. Differences in EQ5D-L pain and discomfort subscores from the time of initial SRS to last follow-up for the overall cohort of 116 patients. Circles represent patients tested.

at least when QOL is the principal factor determining a patient's treatment approach. However, the number of brain metastases at the time of SRS did adversely impact QOL in the current study. Many studies have demonstrated the survival benefits of single or fractionated SRS for more than 5 metastases. ${ }^{7,22}$ Although the number of brain metastases at the time of SRS may not significantly constrain procedural feasibility with contemporary SRS platforms, it did appear to negatively impact the QOL of patients studied prospectively in this registry. Further study of the impact of the number of brain metastases on QOL in SRS patients seems warranted. The SRS registry effort is ongoing and the continued accrual of patients will permit further explorative studies into the factors impacting QOL for not only SRS patients with metastatic disease but also those undergoing radiosurgery for more benign indications. While tumor volume and age may impact overall survival, they need not necessarily be construed as factors that predict an appreciable risk for worsening QOL after SRS. ${ }^{5}$

Early use of SRS from the time of initial brain metas- tasis diagnosis was also a predictor of stable or improving EQ5D-L at last follow-up. The implications of this are unclear and could point to a benefit of earlier control of the intracranial disease. Alternatively, some patients may have had SRS delayed in this study due to the need for treatment of systemic disease, and this active systemic disease could adversely impact the QOL of the patients. Further study is required to elucidate the potential underlying mechanisms of this finding.

Additionally, the current study suggests the existence of sex differences in QOL after SRS. Sex differences in QOL for cancer patients have been previously observed and studies frequently adjust for age and sex differences that can impact QOL. ${ }^{12-14}$ While the current findings need to be validated by other studies of brain metastasis patients, the sex differences found herein as related to pain and discomfort may be cause for increased recognition and, if present, medical treatment in men suffering from these problems during the course of their care for brain metastasis. 
Lastly, the study suggests that anxiety subscores at baseline are frequently predictive of anxiety subscores at last follow-up. Thus, despite the frequently successful radiosurgical treatment of the underlying brain metastases, patient anxiety persists and often remains minimally changed during their disease course. In those patients with high EQ5D-L anxiety subscores at the time of SRS, medical management, cognitive behavioral therapy, support groups, and nontherapeutic tools may prove useful for improving this aspect of QOL. ${ }^{1}$

\section{Study Limitations}

The current study suffers from many limitations. While the study accrued at many sites across the country, the sites were not chosen randomly. Thus, site selection may have biased the patient cohort. While sites were encouraged to include EQ5D-L scores on all brain metastasis patients, not all sites were compliant in this regard and patients clinically doing well are more likely to return for followup and complete QOL reassessments. Brain metastasis patients who did not have initial or post-SRS EQ5D-L scores were excluded from the analysis, and this may have introduced a bias. Patients were treated per the standard of care at the respective treating sites. While this may introduce some real-world perspective to the treatment approach, the treatment paradigms for selection, delivery, and follow-up of brain metastasis patients undergoing SRS varied from site to site, and such variations could introduce effects within the study population and endpoints.

The use of EQ-5D as a QOL metric for SRS brain metastasis patients is common and has been recently demonstrated by Miller et al. (2017) and Zindler et al. (2017).9,23 However, other QOL metrics, such as the EORTC-BM22, could be utilized to study patients. Also, specific neurocognitive assessments of patients were lacking in the registry-based study but warrant consideration in this patient population.

As the current study was intended only to evaluate preSRS attributes and their impact on QOL, we did not assess post-SRS parameters that may impact QOL, such as adverse SRS effects (e.g., necrosis), local or distant intracranial disease progression, systemic disease progression, and need for further procedures. These parameters will be assessed in future registry-based studies.

The effect of number of brain tumors and lower QOL may be linked to systemic disease progression or need to change systemic therapy. Unfortunately, this information is not complete for many patients and, therefore, the interactions between these variables and QOL were not evaluated. Lastly, the study time period was selected based upon statistical guidance that a sufficiently powered cohort of brain metastasis patients had been achieved. However, many of these patients remain alive and part of the registry for which additional patient data will be accrued. Thus, unlike with a fixed endpoint to a clinical trial, additional changes in QOL as a function of time will need to be explored in this registry-based study.

\section{Conclusions}

In brain metastasis patients, earlier treatment with SRS after diagnosis of brain metastasis led to improved QOL, and an increased number of tumors at presentation predicted a decrease in QOL. Men appear to be at greater risk for poor QOL associated with pain and discomfort. Anxiety at the time of SRS appears to predict anxiety at last follow-up, and those with high anxiety at baseline may benefit from approaches to mitigate this detrimental factor in their overall QOL.

\section{References}

1. Bates GE, Mostel JL, Hesdorffer M: Cancer-related anxiety. JAMA Oncol 3:1007, 2017

2. Bottomley A, Flechtner H, Efficace F, Vanvoorden V, Coens $\mathrm{C}$, Therasse P, et al: Health related quality of life outcomes in cancer clinical trials. Eur J Cancer 41:1697-1709, 2005

3. Bragstad S, Flatebø M, Natvig GK, Eide GE, Skeie GO, Behbahani M, et al: Predictors of quality of life and survival following Gamma Knife surgery for lung cancer brain metastases: a prospective study. J Neurosurg 129:71-83, 2018

4. Chang EL, Wefel JS, Hess KR, Allen PK, Lang FF, Kornguth DG, et al: Neurocognition in patients with brain metastases treated with radiosurgery or radiosurgery plus whole-brain irradiation: a randomised controlled trial. Lancet Oncol 10:1037-1044, 2009

5. Emery A, Trifiletti DM, Romano KD, Patel N, Smolkin ME, Sheehan JP: More than just the number of brain metastases: evaluating the impact of brain metastasis location and relative volume on overall survival after stereotactic radiosurgery. World Neurosurg 99:111-117, 2017

6. EuroQol Group: EuroQol-a new facility for the measurement of health-related quality of life. Health Policy 16:199208, 1990

7. Hara W, Tran P, Li G, Su Z, Puataweepong P, Adler JR Jr, et al: Cyberknife for brain metastases of malignant melanoma and renal cell carcinoma. Neurosurgery 64 (2 Suppl):A26A32, 2009

8. Liang KY, Zeger SL: Longitudinal data analysis using generalized linear models. Biometrika 73:13-22, 1986

9. Miller JA, Kotecha R, Barnett GH, Suh JH, Angelov L, Murphy ES, et al: Quality of life following stereotactic radiosurgery for single and multiple brain metastases. Neurosurgery 81:147-155, 2017

10. Nayak L, Lee EQ, Wen PY: Epidemiology of brain metastases. Curr Oncol Rep 14:48-54, 2012

11. Pan HC, Sun MH, Chen CC, Chen CJ, Lee CH, Sheehan J: Neuroimaging and quality-of-life outcomes in patients with brain metastasis and peritumoral edema who undergo Gamma Knife surgery. J Neurosurg 109 Suppl:90-98, 2008

12. Pashos CL, Flowers CR, Kay NE, Weiss M, Lamanna N, Farber C, et al: Association of health-related quality of life with gender in patients with B-cell chronic lymphocytic leukemia. Support Care Cancer 21:2853-2860, 2013

13. Pickard AS, Jiang R, Lin HW, Rosenbloom S, Cella D: Using patient-reported outcomes to compare relative burden of cancer: EQ-5D and Functional Assessment of Cancer TherapyGeneral in eleven types of cancer. Clin Ther 38:769-777, 2016

14. Pickard AS, Wilke CT, Lin HW, Lloyd A: Health utilities using the EQ-5D in studies of cancer. Pharmacoeconomics 25:365-384, 2007

15. Sheehan JP, Kavanagh BD, Asher A, Harbaugh RE: Inception of a national multidisciplinary registry for stereotactic radiosurgery. J Neurosurg 124:155-162, 2016

16. Skeie BS, Eide GE, Flateb $\varnothing$ M, Heggdal JI, Larsen E, Bragstad S, et al: Quality of life is maintained using Gamma Knife radiosurgery: a prospective study of a brain metastases patient cohort. J Neurosurg 126:708-725, 2017 
17. Soffietti R: Reply to M.C. Chamberlain and C. Gemici. J Clin Oncol 31:2636-2637, 2013

18. Soffietti R, Kocher M, Abacioglu UM, Villa S, Fauchon F, Baumert BG, et al: A European Organisation for Research and Treatment of Cancer phase III trial of adjuvant wholebrain radiotherapy versus observation in patients with one to three brain metastases from solid tumors after surgical resection or radiosurgery: quality-of-life results. J Clin Oncol 31:65-72, 2013

19. Soon YY, Tham IW, Lim KH, Koh WY, Lu JJ: Surgery or radiosurgery plus whole brain radiotherapy versus surgery or radiosurgery alone for brain metastases. Cochrane Database Syst Rev (3):CD009454, 2014

20. Trifiletti DM, Lee CC, Kano H, Cohen J, Janopaul-Naylor $\mathrm{J}$, Alonso-Basanta M, et al: Stereotactic radiosurgery for brainstem metastases: an international cooperative study to define response and toxicity. Int J Radiat Oncol Biol Phys 96:280-288, 2016

21. Trifiletti DM, Sheehan JP, Grover S, Dutta SW, Rusthoven CG, Kavanagh BD, et al: National trends in radiotherapy for brain metastases at time of diagnosis of non-small cell lung cancer. J Clin Neurosci 45:48-53, 2017

22. Yamamoto M, Serizawa T, Shuto T, Akabane A, Higuchi Y, Kawagishi J, et al: Stereotactic radiosurgery for patients with multiple brain metastases (JLGK0901): a multi-institutional prospective observational study. Lancet Oncol 15:387-395, 2014

23. Zindler JD, Bruynzeel AME, Eekers DBP, Hurkmans CW, Swinnen A, Lambin P: Whole brain radiotherapy versus stereotactic radiosurgery for 4-10 brain metastases: a phase III randomised multicentre trial. BMC Cancer 17:500, 2017

\section{Disclosures}

The registry effort was supported by grant funding to NPA from BrainLab, Elekta, AANS, and ASTRO. Individual sites received nominal grant funding from NPA. Drs. Sheehan and Kondziolka serve as representatives for NPA.

Dr. Grills reports having stock ownership in and serving on the Executive Board of Directors of Greater Michigan Gamma Knife and receiving funding for non-study-related research from Elekta through her institution.

\section{Author Contributions}

Conception and design: Sheehan, Kavanagh. Acquisition of data: Sheehan, Chiang, Warnick, Kondziolka, Kavanagh. Analysis and interpretation of data: Sheehan, Grills, Kavanagh. Drafting the article: Sheehan. Critically revising the article: Sheehan, Grills, Chiang, Berg, Warnick, Kondziolka, Kavanagh. Reviewed submitted version of manuscript: Sheehan, Kavanagh. Approved the final version of the manuscript on behalf of all authors: Sheehan. Statistical analysis: Dong, Berg. Administrative/technical/material support: Sheehan. Study supervision: Sheehan, Grills, Kavanagh.

\section{Correspondence}

Jason P. Sheehan: University of Virginia Health System, Charlottesville, VA.jps2f@virginia.edu. 